\title{
AUtONET: INTER-VeHICle COMMUNICATION AND NETWORK VEHICULAR TRAFFIC
}

\author{
Will Recker ${ }^{1}$, Wen-Long Jin ${ }^{2}$, Xu Yang ${ }^{2}$ and James Marca ${ }^{2}$
}

\begin{abstract}
With the proliferation of wireless communication technologies, inter-vehicle communication (IVC) could potentially be applied to solve ever-worsening transportation problems around the world. In this paper, we study impacts of network vehicular traffic on IVC, apply IVC to route-guidance, and report preliminary field tests. These studies are intended to demonstrate the feasibility of IVC-based traveler information system and the interaction between IVC and network vehicular traffic.
\end{abstract}

\section{INTRODUCTION}

Federal, state, and local public expenditures on transportation have exceeded \$100 billion annually over the last decade; another $\$ 580$ billion annually is spent on purchasing, operating, and maintaining private household vehicles. Even so, urban freeway and arterial networks are managed based on only the coarsest of information: sparsely distributed inductance loop detectors buried in traffic lanes transmit single bits of data indicating the presence of a vehicle over wires to a traffic control center. Within the automotive telematics industry, currently all commercialized traffic information systems are centralized systems, either integrated within an in-vehicle navigation system or as a stand alone system. These systems all require a centralized traffic information center (TIC) to process traffic information typically derived from data collected from fixed detection stations installed in the roadways connected to the TIC via wired cable or from "floating-car" data polled from vehicles connected to the TIC via wireless modem, and then the processed data distributed to their users via wired or wireless communication connections. Shortcomings of these centralized information systems include: heavy capital investment is needed to initiate the system, difficulty of system upgrade, vulnerability to system failures, and general lack of specific relevancy of information provided to any particular trip.

With the development of wireless communication and information technologies and ubiquitous existence of wireless communication devices, Advanced Transportation Information System (ATIS) based on inter-vehicle communication (IVC) are not only possible but probable. A number of efforts are currently underway to investigate intervehicle communications based on mobile ad hoc networking technology as a means of developing "internet on the road" (e.g. CarTALK 2000; FleetNet, Franz et al, 2001). However, to date, these efforts have focused more on the protocols and routing

\footnotetext{
${ }^{1}$ Department of Civil and Environmental Engineering, Institute of Transportation Studies and California Institute for Telecommunications and Information Technology, University of California, Irvine, CA 92697, USA. Email: wwrecker@uci.edu. Corresponding author

${ }^{2}$ Institute of Transportation Studies and California Institute for Telecommunications and Information Technology, University of California, Irvine, CA 92697, USA.
} 
algorithms for information transmissions than on the application of the technology as the foundation for a decentralized, real-time traffic information system. In today's market, radio transmitters/receivers are becoming cheaper and smaller and, although they may not meet requirements of such safety-critical applications as collision avoidance and automated driving, they can be used in less critical applications falling under the domain of advanced traveler information systems (ATIS) and management systems (ATMS). Recently, the potential for distributed traffic information systems based on IVC has gain interest in the transportation academic community (Ziliaskopoulos and Zhang, 2003).

To explore the advantages of IVC in developing Intelligent Transportation Systems (ITS), researchers at the California Institute of Telecommunications and Information Technology and the Institute of Transportation Studies of the University of California, Irvine, have been engaged in a comprehensive research effort aimed at the development of an autonomous, self-organizing, transportation management, information, and control system. Called Autonet, the effort has the long-term goal of deployment of an autonomous, self-organizing information network for effective management of interactions among intelligently informed vehicles, roadways, stations and consumers (drivers). The Autonet concept proposes to use vehicle-to-vehicle and vehicle-toinfrastructure communications to leverage cooperative, vehicle-centric pervasive computing as a platform for transportation management. At its core, Autonet can be visualized as a bundle of services supporting an arbitrary collection of transportation management applications. These services are provided by accessing a decentralized collection of computer systems using a variety of protocols, which in turn are implemented on any number of physical networking architectures. In contrast to centralized systems, distributed traffic information systems based on information exchange among vehicles do not require any public infrastructure installed in the network; rather, they rely only on on-board devices installed in at least some vehicles traversing the roadway network. Because such systems are totally independent of public infrastructure, they will be market-driven and self-maintained, without the noted shortcomings of centralized systems. Vehicles in the traffic network generate information, exchange information, process information, and distribute information; they are not only the users (passive beneficiaries) of the system, but also information sources (active contributors) to the system.

Autonet also poses significant challenges compared to infrastructure-based networks: (1) the routing problem, which is already hard to solve in fixed networks, is substantially more complicated in such a mobile ad-hoc network as Autonet; (2) although rapid progress made toward higher data transmission rates based on the IEEE 802.11 standard for wireless local area networking (WLAN) has put the promise of truly mobile computing within reach, communications bandwidth remains a scarce resource in a mobile ad-hoc network due to signal interference; (3) the possibility of propagation of malicious misinformation and access to any particular traveler's origin-destination information can pose a serious security problem.

Then, what kinds of communication technologies are required for Autonet to benefit the management and control of network vehicular traffic? To answer this question, which is important for developers of both communication technologies and ITS strategies, we have to understand the fundamental performance of such a complex system as Autonet, in 
which transportation and communication systems are coupled with each other. In Section 2, we discuss how network vehicular traffic can affect the performance of IVC. In Section 3, we discuss how traffic information relayed through IVC can benefit the performance of traffic system. In Section 4, we discuss preliminary field test of Autonet. These discussions can further our understanding of an Autonet system and shed some light on the development of efficient communication technologies for Autonet applications.

\section{Impacts of Network Vehicular Traffic on the Prospects for IVC-Based ATIS}

IVC is highly coupled to network vehicular traffic, and characteristics of the latter can have important impacts on the former. The "information wave" resulting from instantaneous uni-directional and bi-directional information propagation via peer-to-peer information exchange among IVC-equipped vehicles in the network (Jin and Recker, 2004) and non-instantaneous bi-directional information propagation (Ziliaskopoulos and Zhang 2003) in linear traffic networks under both incident-free and incident conditions have been studied using analytical approaches. Network vehicular traffic systems are characterized by vehicles' mobility, i.e., potentially high fluctuations in relative positions of any two vehicles. Such high mobility of vehicles can result in a highly dynamic topology of IVC (Rudack et al., 2002), which yields short-lived communication paths and other deficiencies (Blum, et al, 2004). When the market penetration rate is relatively high, an efficient approach to propagating information is through multihop broadcasting. For example, Hartenstein, et al (2001) studied the probability for establishing connection between two equipped vehicles through multihop broadcasting with a market penetration rate of $10 \%$, using a cellular automaton model to simulate vehicle movements. Conversely, when the market penetration rate is relatively low, as would be expected in the early phases of deployment of IVC systems, such multihop broadcasting would likely not be very successful in maintaining a useful information chain. Rather, the vehicle movements themselves would likely be the prominent mechanism for establishing a chain.

\subsection{The Potential for Multihop Broadcasting}

We argue that that under market penetration conditions that support multihop broadcasting it is reasonable to assume the information propagation to be instantaneous with respect to vehicle movement, based on the observations that traffic information messages are usually short and modern communication devices have large communication bandwidth. For example, a packet of 73 bytes can be transmitted once in 110 ms with a communication bandwidth of $3.6 \mathrm{~kb} / \mathrm{s}$ (Briesemeister et al., 2000); i.e., it takes only $1.1 \mathrm{~s}$ for information to reach as far as $5 \mathrm{~km}$ with a communication range of $500 \mathrm{~m}$. Alternatively, the maximum change in the relative positions of vehicles in the same or opposition directions is about $73 \mathrm{~m}$ (with a relative speed of $240 \mathrm{~km} / \mathrm{h}$ ). The assumption of instantaneity is increasingly valid as traffic becomes congested, where vehicle speed is significantly lower.

The irrelevance of vehicle mobility can be demonstrated by Figure 1 for a uniform traffic stream, which shows the connectivity of two vehicles at certain distances for four 
different transmission ranges. In this figure, one group of curves with marks are from Hartenstein, et al (2001), which simulates vehicle mobility with a traffic simulator; the other group without marks are from an analytical model in Jin and Recker (2005), which assumes instantaneous information propagation in IVC system. The comparison shows that these two groups of results are very close to each other. In this sense, vehicle mobility is almost irrelevant to connectivity of IVC system.

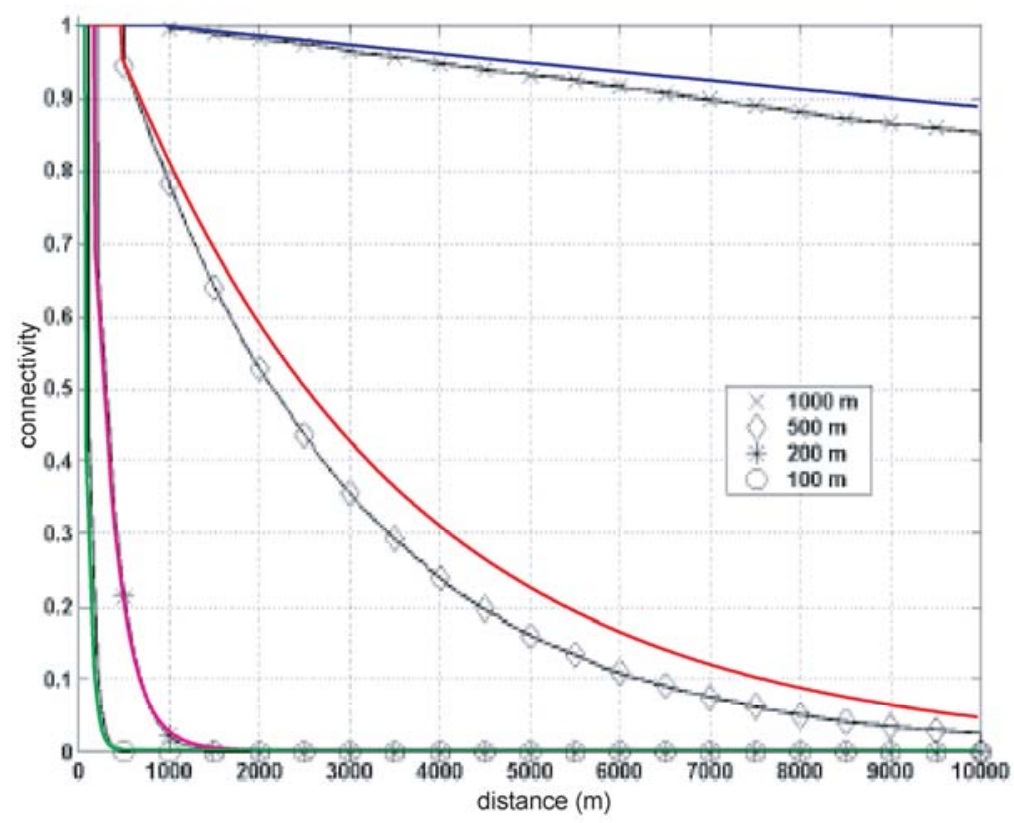

Figure 1. Comparison of multihop connectivity in an IVC system with and without considering vehicle mobility: traffic density $=58 \mathrm{veh} / \mathrm{km}$, market penetration rate $=10 \%$; lines with marks are from Figure 1 of Hartenstein, et al (2001), the corresponding solid lines are from an analytical model of instantaneous multihop connectivity in Jin and Recker (2005).

These results support the conclusion that, while vehicle mobility may be a relevant factor in information propagation by IVC when the market penetration rate is low, it has little impact on connectivity properties for a relatively high market penetration rate.

In most studies of wireless communication networks, communication nodes are assumed to follow Poisson distribution. That is, locations of communication nodes are independent and uniformly randomly distributed on a plane or line. However, vehicles in a road network usually do not follow spatial Poisson distribution due to inter-dependence of vehicle positions. The existence of traffic patterns can be shown by a traffic stream subject to restrictions of road geometry, traffic signals, and car-following rules. For example, in the vicinity of a lane-drop location or merging junction, traffic is usually more congested on the upstream part; gaps between platoons of vehicles on surface streets constantly change due to traffic signal controls; shock waves formed by the closure of a lane due to an incident typically result greater vehicle density downstream of the shock wave interface. Since these situations are precisely those for which real-time 
traffic information can be beneficial, it is important to analyze such non-Poisson distribution patterns of vehicles on a road both when considering the performance of an IVC system or in determining appropriate technologies to implement an IVC system.

To demonstrate the effect of distribution patterns of vehicles, we consider a traffic stream of 580 vehicles on a link of $10 \mathrm{~km}$, market penetration rate $10 \%$, and transmission range of $500 \mathrm{~m}$. Then we demonstrate the multihop connectivity at different distances for three types of distribution patterns in Figure 2. The solid curve shows connectivity of a uniform traffic stream with density of $58 \mathrm{veh} / \mathrm{km}$, which can be considered as a discrete approximation of spatial Poisson distribution. The dotted curve is for a traffic stream with $38 \mathrm{veh} / \mathrm{km}$ for the upstream half and $78 \mathrm{veh} / \mathrm{km}$ for the downstream half. That is, in the direction of information propagation, the downstream traffic is more congested, and this situation resembles a shock wave. The dashed curve is for a traffic stream with congested upstream: $78 \mathrm{veh} / \mathrm{km}$ for the upstream half and $38 \mathrm{veh} / \mathrm{km}$ for the downstream half. This situation can occur when there is a lane-drop at $5 \mathrm{~km}$. From this figure, we can see that different distribution patterns generate different connectivity profiles. Since different traffic patterns are very common in real vehicular traffic, it is essential to analyze their effects on IVC.

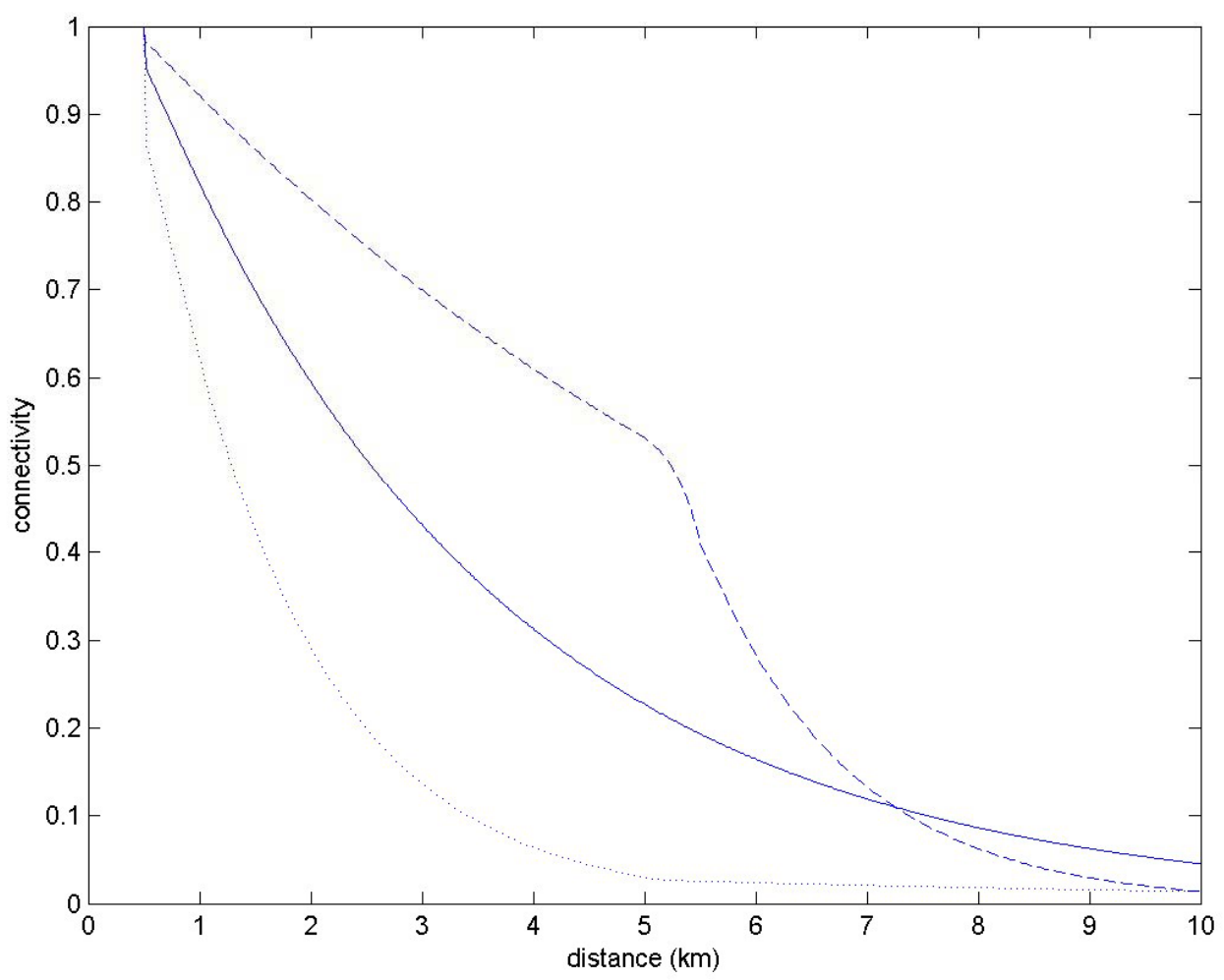

Figure 2. The effect of distribution patterns of vehicles on multihop connectivity

The impact of the location of information source relative to these discontinuities and that of the size of gaps are discussed in Jin and Recker (2006) and Jin and Recker (2005). The 
results of microscopic simulation studies conducted by Yang and Recker (2006a) generally support the theoretical results reported in Jin and Recker (2005).

\subsection{The Potential for Vehicle-dynamic Broadcasting under Low Market Penetration}

Vehicle movements can be beneficial for propagating information throughout a road network, particularly when the market penetration rate of equipped vehicles is very low at the initial stage and there are relatively few equipped vehicles within the communication range in the same traveling direction as an information source. Rather, the primary mechanism for information transfer is to wait for an equipped vehicle coming from the opposite direction to carry a message to equipped vehicles further upstream.

In this case, information flow is aided by a rapidly-changing mix of vehicles passing the target vehicle in the opposite direction, all of which are within IVC communication radius range, if only for a brief time. Thus, in the bi-directional propagation case, the threshold of IVC equipment market penetration rate and vehicle density in the network to support the inter-vehicle information system is expected to be much lower compared to the multihop propagation cases. This is a particularly important attribute of the traffic system since having a relatively low threshold for IVC equipment market penetration rate required to support such a decentralized and self-organizing system is critical to evolving the system. Here the two flows of traffic, moving in opposite directions, effectively increase the vehicle density in the time domain; traffic flow actually helps, rather than hinders, information flow to accelerate its propagation.

For IVC communication radius range of $50 \mathrm{~m}$, which is the smallest value used in our simulation studies, and IVC market penetration rate of 0.01 , which is the smallest value tested in (Yang and Recker, 2006a), although the probability of successful IVC between any two vehicles is found to be much less than 1 , the traffic information propagation distance (Figure 3) is much longer than the communication radius range, making information flow possible in the traffic network. All results found imply that it is quite possible that a vehicle-to-vehicle information sharing based traffic information system could be functional at the low market penetration rate (less than 0.02) even if the IVC technology only allows neighboring IVC-equipped vehicles exchange information when the distance between them is less than 50 meters. 


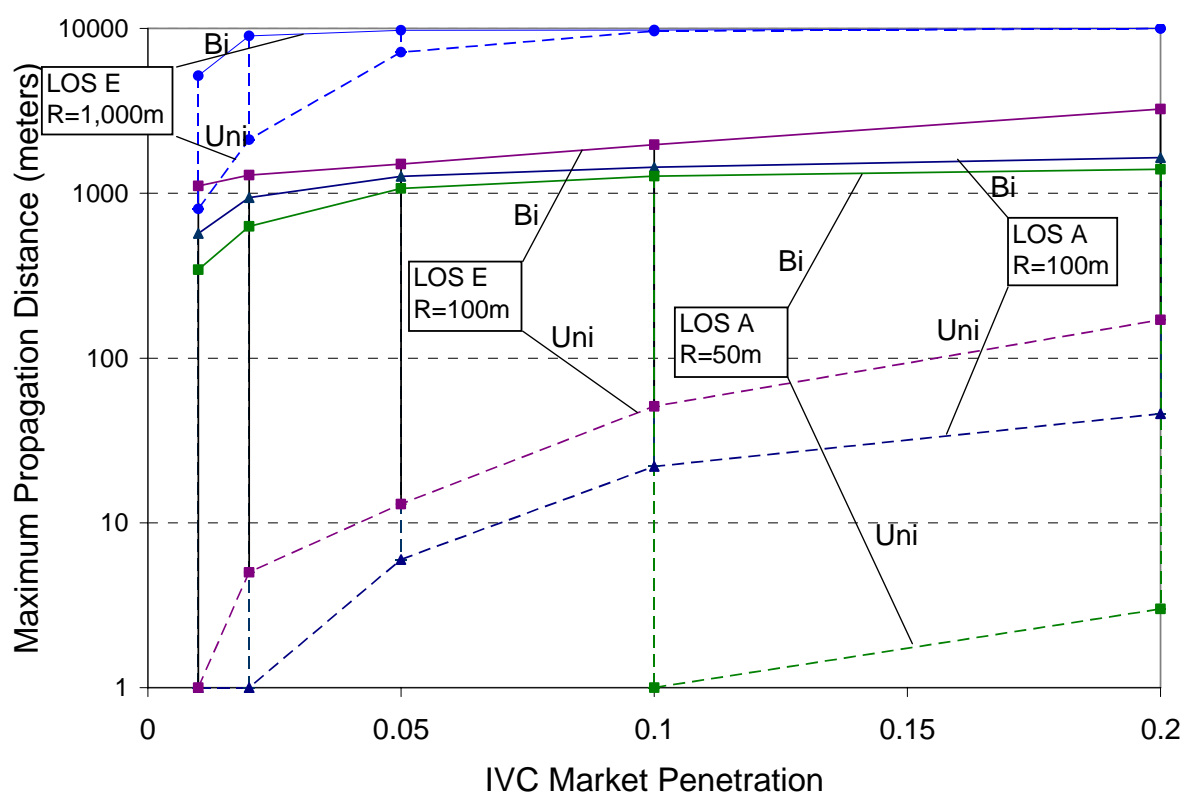

Figure 3. Maximum expected propagation distance under bi-directional communication (Yang and Recker, 2006a)

Under incident conditions, as shown in Figure 4, for example, in which the propagation distance differences between the incident information wave and traffic shock wave are plotted for various levels of market penetration (MPR), communications range (R), and traffic density in the freeway lanes opposite to the incident (XLOS), the values of propagation distance differences for incident information wave and traffic shock wave are found to be significantly greater than zero for IVC communication radius range as small as 50 meters and marker penetration rate as low as 0.01 .

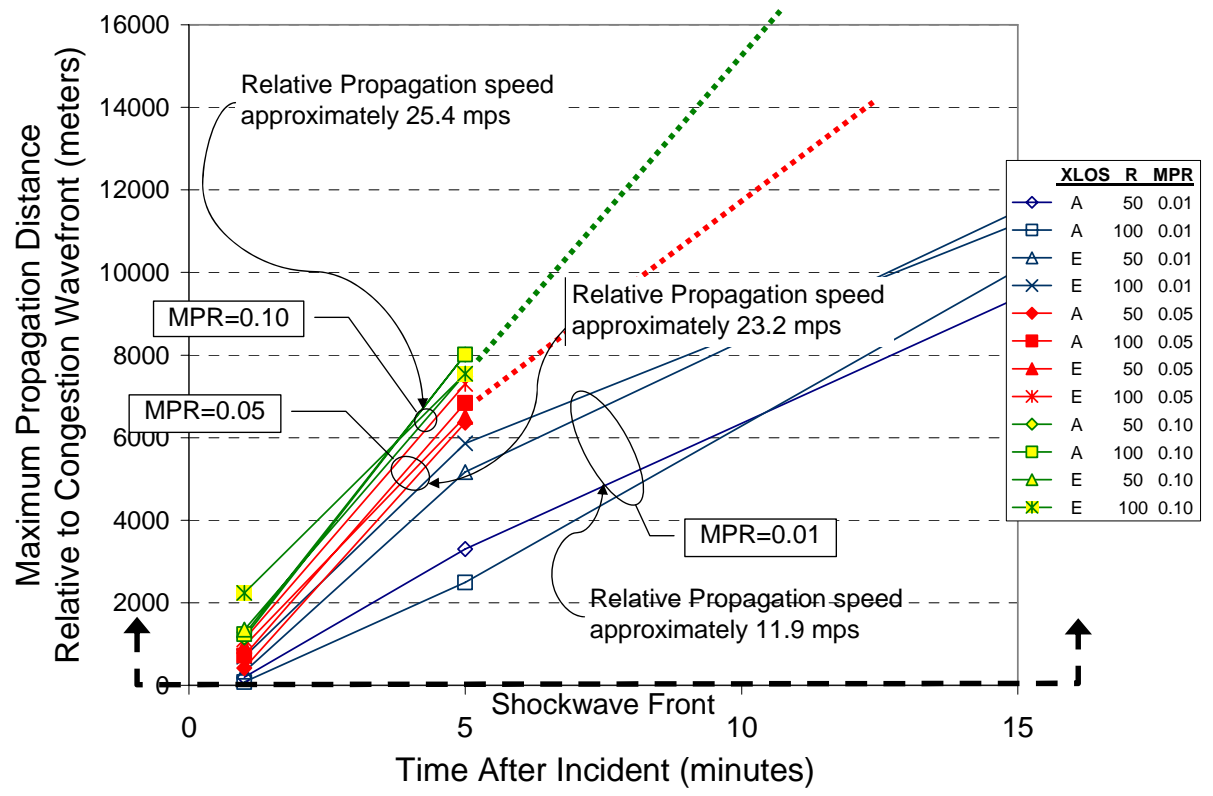


Figure 4. Difference between information wave front and traffic shockwave front (Moderate Traffic LOS C) (Yang and Recker, 2006a)

The two opposite traffic flows not only effectively increase the vehicle density in the time domain, but also allow IVC vehicles in the direction of the roadway impacted by the incident to get incident information through IVC vehicles in the other direction of the roadway not impacted by the incident. In this way, traffic flow helps information flow to accelerate its propagation speed under incident conditions.

For conditions illustrated in Figure 4 (i.e., relatively low market penetration coupled with relatively short communications range), the relative speed of propagation of the incident information depends on the directional traffic conditions (LOS and XLOS), as reflected by the probability of successful communication between two vehicles. The speed itself is influenced both by any delay in the observation of the incident (e.g., caused by the chance arrival of an IVC-capable vehicle in the opposite lanes of traffic) and the actual propagation of the information via either multihop along the traffic queued behind the incident or through the "carrying" of the information upstream by vehicles in the opposing lanes of traffic (or a combination of the two). For combinations of low market penetration rates and light traffic in the opposing lanes, the information packet propagation is mostly affected by the probability of successful IVC. At sufficiently high levels of the probability of success (e.g., combinations of high market penetration, large communications radius, and dense traffic), information propagation is mainly by multihop IVC which should be virtually instantaneous (limited only by IVC technology); otherwise vehicle movement in opposite direction should be the primary mechanism to pass along the incident information packet (limited by the speed of the opposing traffic, i.e., 50-70 mph). Simulation results indicate that, over a very broad range of LOS and XLOS, a threshold value of the probability of successful transmission between any two vehicles of approximately 0.9 is required before the speed of information propagation becomes virtually instantaneous - at probabilities less than this threshold, information propagation speed take on relatively stable values centered about the speed of the opposing traffic stream itself.

\section{The Potential Value of IVC as an ATIS}

Does access to such incident information produce any measurable effect on travel time savings to those who receive the information, or to the network as a whole? As shown in (Yang and Recker, 2006b), the simulation results hold significant promise for the efficacy of the system. As an example, results in Figure 5 are for a 5,000m $\times 5,000 \mathrm{~m}$ grid network with $1,000 \mathrm{~m}$ blocks, for the case of an incident at the center of the network and uniform origin-destination pattern. Shown in the figure are travel time savings for the whole traffic system, including travelers with and without IVC capability. Beyond a threshold value of approximately $1 \%-2 \%$ for market penetration rate, total vehicle travel time decreases with increasing penetration and communications range for all traffic demand levels considered. This is an outcome of the IVC-capable vehicles' travel time savings from re-routing and the travel time savings for non-IVC vehicles whose paths include the incident link (now with less demand as a result of IVC-capable vehicles rerouting) being greater than the increased travel time on the links comprising the IVCcapable vehicles' re-routing paths. 


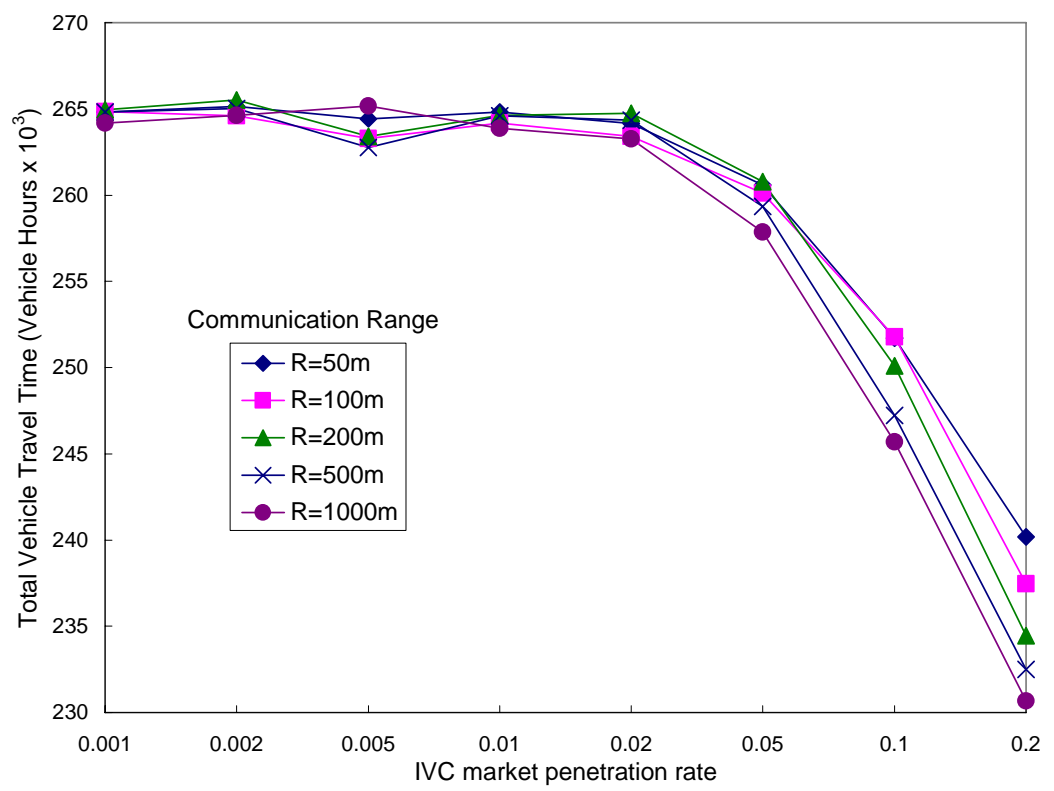

Figure 5. Impact of IVC on total system travel time (Arterial grid with moderate traffic) (Yang and Recker, 2006b)

Similar results have been obtained for the case of freeways. Figure 6 gives an example of total system performance along a $20 \mathrm{~km}$ stretch of an 8-lane freeway and parallel 4-lane arterial connected by on/off ramps spaced at 2000-meter intervals, as measured by the vehicle travel time for all vehicles potentially impacted by an incident.

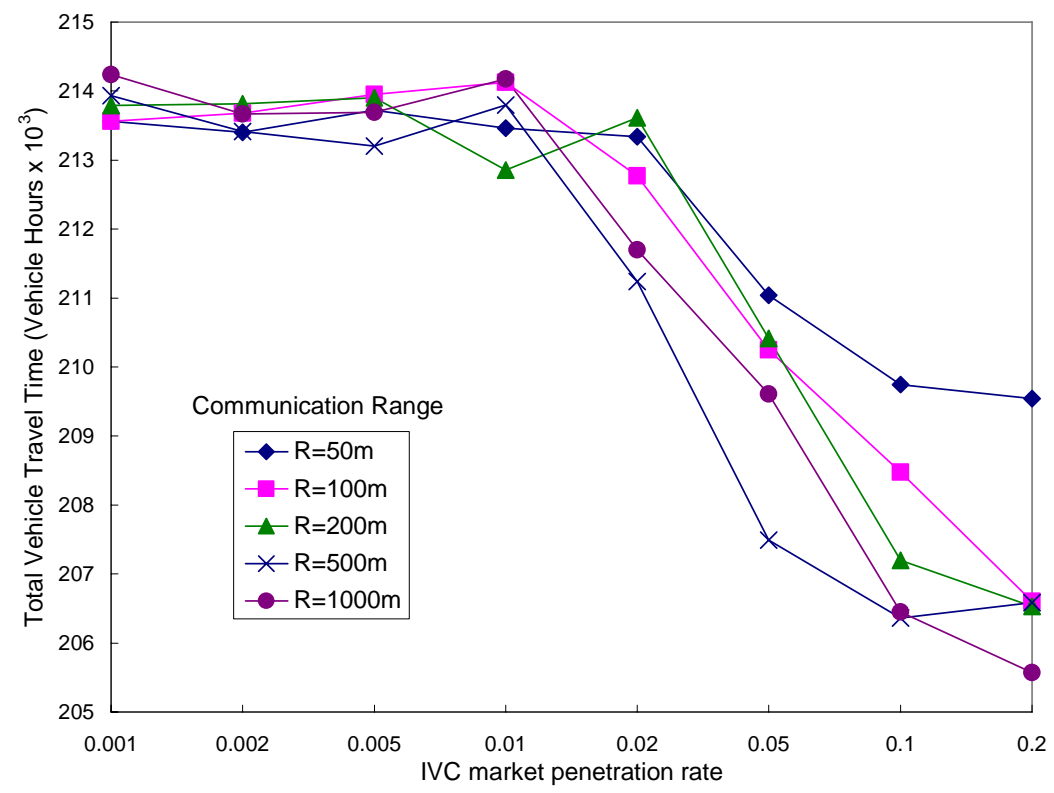

Figure 6. Impact of IVC on total system travel time (Freeway with moderate traffic) (Yang and Recker, 2006b) 


\section{Preliminary Field Test of Autonet}

An in-vehicle client with an informative GUI has been implemented a preliminary implementation of some of the key Autonet concepts. This client continuously listens for other clients, and exchanges knowledge about network incidents once contact is made. The results indicate that knowledge about traffic conditions can be propagated successfully using this system. The client programs were also used to test the actual throughput possible for messages sent from one vehicle to another using $802.11 \mathrm{~b}$ wireless hardware. These measurements establish the maximum throughput at about 4,000 incidents for two vehicles moving in opposite directions at highway speeds.

The results document the current capabilities of hardware for vehicle-to-vehicle communication of traveler information, provide working protocols and communications parameters for improving future large-scale simulation studies, and give us an in-vehicle client application which will be used in the future for studies of driver interaction and market acceptance.

In addition, a variety of benchmarking tests were run that were created for testing the performance of 802.11b technology as it applies to Autonet. The table below shows the result of this effort. We collected all of these measurements with a Lucent Technologies ORiNOCO 802.11b network adapter connected to a $5.5 \mathrm{dBi}$ external antenna.

Vehicle Speed (mph)

Benchmark Measurement

Number of incident information packets exchanged by two vehicles passing in opposite directions

Time two vehicles, passing at identical speed in opposite directions, can stay within signal range (seconds)

Round-trip time for message passing between two vehicles traveling at the same speed in opposite directions (milliseconds)
40 55 70 5,996 4,295 3,566 18 10 71 80 81

The first measurement shows the results of tests of the ability of $802.11 \mathrm{~b}$ to transfer incidents between two Autonet-enabled vehicles that pass each other in opposite directions at identical speeds. Shown in the table are the average number of incidents that each vehicle could send vs. speed of each vehicle (not relative speed). At $40 \mathrm{mph}$, for example, a total of nearly 12,000 incidents on average was sent and received on each single pass.

As expected, the number of incidents exchanged drops sharply as the speed of the vehicles increases. The decline is largely due to the shorter time in which the two vehicles are within signal range, but we suspect that the Doppler effect also plays a role 
in limiting throughput with increasing speed. We note, however, that even when two cars travel at highway speed--70 miles per hour-they are able to send and receive over 3,000 incidents each. These are very promising results, as they show that one vehicle can inform another about every single segment of road for a typical urban area of 64 square kilometers. If we assume that one out of every five segments is an incident, the area grows to 324 square kilometers.

The second measurement displays the amount of time during which two vehicles, when traveling in opposite directions, could remain in $802.11 \mathrm{~b}$ signal range of each other and successfully exchange incidents. We collected these measurements with the help of an external antenna; this explains the surprisingly long periods during which the two vehicles were able to remain in contact; we were unable to exchange incidents reliably without the antenna, even at slow speeds. The consequence for Autonet is that any production version of our prototype would require an external antenna.

The final measurement shows the round-trip time for message exchange using 802.11b. Here, we define "round-trip time" as the time required for one vehicle to send an incident request to another and fully receive a response to the query. Based on these measurements, we calculate that when using a request/response method of incident exchange, approximately 200 incidents can be received per vehicle.

This number is an order of magnitude lower than the number of incidents a vehicle is able to receive when using the broadcast method of incident exchange. This is one reason why we have focused on the broadcast method of exchange for our Autonet prototype. On the other hand, the number of messages that need to be passed between any two vehicles is a function of the number of Autonet-compliant vehicles, and the level of system awareness that is desired and possible. If a vehicle has one thousand unique incidents, but $90 \%$ of them are old, then 100 messages is enough. Alternately, if two platoons of ten cars each are passing each other, and if each car is responsible for disseminating some fragment of the platoon's collective knowledge, then 100 messages per car is also probably enough. Finally, there is also the possibility to develop a hybrid messaging protocol, in which the usual request/ acknowledgment messages are followed by a broadcast period. This hybrid method may prove to be the best solution. These questions need to be explored with further research and development.

\section{CURRENT WORK}

Analytical and simulation studies both verify that IVC can evolve as an effective ATIS. The prototype version of an Autonet computer has successfully demonstrated that the basic Autonet idea is sound - that vehicles can be expected to share information about traffic conditions in a real-world environment. In addition, the exercise of turning theory into reality has shown several areas which need improvement. Chief among these are deficiencies in the way novel information is detected and shared with the driver and with other Autonet peers. For the former, we are actively researching Bayesian inference techniques as in Liao et al. (2004), which will help determine what information is relevant to the current trip. Improving and expanding the Autonet middleware is another area of active development, which will allow decentralized group coordination of event detection, measurement, and description. In order to address questions related to 
scalability as penetration rates increase, we are continuing the Autonet simulation studies. Finally, there is no requirement that Autonet peers be vehicles. Specifically, we will be developing prototype devices for pedestrians and for roadside nodes, with the latter allowing traffic authorities to tap into the knowledge available in the Autonet to improve signal timing and coordination.

\section{REFERENCES}

Blum, J.J., A. Eskandarian, and L.J. Hoffman. Challenges of intervehicle ad hoc networks. IEEE Transactions on ITS, 5(4):347-351, 2004.

Briesemeister, L., L. Schafers, and G. Hommel. Disseminating messages among highly mobile hosts based on inter-vehicle communication. In IEEE Intelligent Vehicles Symposium, pages 522-527, 2000.

CarTALK 2000. Safe and comfortable driving based upon inter-vehicle communication. http://www.cartalk2000.net/. Accessed November 11, 2003.

Franz, W., Eberhardt, R. and Luckenbach T., 2001, FleetNet - Internet on the Road, 8th World Congress on Intelligent Transportation Systems, ITS 2001, Sydney, Australia, October 2001

Hartenstein, H., B. Bochow, A. Ebner, M. Lott, M. Radimirsch, and D. Vollmer. Position-aware ad hoc wireless networks for inter-vehicle communications: the fleetnet project. In Proceedings of the 2nd ACM international symposium on Mobile ad hoc networking \& computing, pages 259 - 262, Long Beach, CA, USA, 2001.

Jin, W.-L. and W. W. Recker. Instantaneous information propagation in a traffic stream through inter-vehicle communication. Transportation Research Part B, 40(3):230-250, 2006.

Jin, W.-L. and W. W. Recker (2005). An analytical model of multihop connectivity of inter-vehicle communication systems. Submitted to IEEE Transactions on Wireless Communications, 2005.

Liao, L., D. Fox, and H. Kautz. Learning and inferring transportation routines. In Proceedings of the National Conference on Artificial Intelligence(AAAI-04), pages 348-353, Menlo Park, CA, 2004. AAAI Press. URL http://www. cs.washington.edu/ai/Mobile_Robotics/projects/postscripts/gps-aaai-04.pdf. Made available by the author on the Internet.

Rudack, M., M. Meincke, and M. Lott. On the dynamics of ad hoc networks for inter vehicle communications (IVC). In The 2002 International Conference on Wireless Networks (ICWN'02), Las Vegas, Nevada, USA, 2002.

Yang, X. and W.W. Recker. Simulation Studies of Information Propagation in a Selforganizing Distributed Traffic Information System. Forthcoming in Transportation Research Part C, 2006a. 
Yang, X. and W.W. Recker. Modeling dynamic vehicle navigation in a self-organizing, peer-to-peer, distributed traffic information system. Forthcoming in Journal of Intelligent Transportation Systems, 2006b.

Ziliaskopoulos, A. K. and Zhang, J., A Zero Public Infrastructure Vehicle Based Traffic Information System, 2003, TRB Annual Meeting, Washington, D.C. 